\title{
An ideal-valued cohomological index theory with applications to Borsuk-Ulam and Bourgin-Yang theorems
}

\author{
EDWARD FADELL AND SUFIAN HUSSEINI \\ Department of Mathematics, The University of Wisconsin-Madison, Madison, \\ WI 53706, USA
}

\begin{abstract}
Numerical-valued cohomological index theories for $G$-pairs $(X, A)$ over $B$, where $G$ is a compact Lie group, have proved useful in critical point theory and in proving Borsuk-Ulam and Bourgin-Yang theorems. More information (which is lost in taking numerical values) is obtained using an ideal-valued theory, and this theory is applied to estimating the size of the zero set of a $G$-map from certain $G$-manifolds to a $G$-module. Parametrized versions of these theorems are also obtained by a principle which applies quite generally.
\end{abstract}

\section{Introduction}

Ljusternik-Schnirelmann category and cohomological index theory (see e.g. [4], [5] and [6]) are both examples of numerical-valued 'index theories' which are quite useful in proving 'Borsuk-Ulam' and 'Bourgin-Yang' theorems as well as in the study of critical points of invariant functionals. Our objective here is to begin the study of an ideal-valued index theory which has distinct advantages over its numerical counterparts. In the simplest situation, if $X$ is a $G$-space, Index ${ }^{G} X$ is an ideal in the ring $\Lambda=H^{*}(B G ; \mathbb{K})$, where $\mathbb{K}$ is an appropriate coefficient field. The corresponding numerical index $\operatorname{dim}_{\kappa}\left(\Lambda /\right.$ Index $\left.{ }^{G} X\right)$ can be infinite (hence of limited value) even when Index ${ }^{G} X$ is a proper ideal conveying useful information. In addition, two $G$-spaces $X$ and $Y$ may be distinguished by the ideals Index ${ }^{G} X$ and Index ${ }^{G} Y$ while the corresponding numerical values are finite and equal. As an illustration, this theory will be applied to the following problem of Lasry and Magill (see [7] for an alternative solution) which arose while they were working on the equilibrium of incomplete markets (mathematical economics). In its unparametrized form it is the following question of the Borsuk-Ulam type. Does an $O(k)$-equivariant map $f: V_{n, k} \rightarrow\left(\mathbb{R}^{k}\right)^{n-k}$ have a non-empty zero set? In this case the numerical index of $V_{n, k}\left(k\right.$-frames in $\left.\mathbb{R}^{n}\right)$ is finite while that of $\left(\mathbb{R}^{k}\right)^{n-k}-0$ is infinite, which puts the problem outside the scope of numerical-valued theory. The technique employed observes first that the existence of a $G$-map $f: X \rightarrow Y$, where $X$ and $Y$ are $G$-spaces, forces Index ${ }^{G} X \supset \operatorname{Index}^{G} Y$. Then one computes the ideals in question and shows the containing relation is false in the case of $V_{n, k}$ and $\left(\mathbb{R}^{k}\right)^{n-k}-0$. The parametrized version considers an $O(k)$-map $f: P \times V_{n, k} \rightarrow P \times\left(\mathbb{R}^{k}\right)^{n-k}$, where $P$ is a parameter space on which $O(k)$ acts trivially. 
Section 2 contains a brief description of index theory of pairs $(X, A)$ over a base $B$ along with basic properties, in the setting of a general multiplicative cohomology theory, and quickly specializes to the index of a single $G$-space employing the $G$-cohomology of Borel. Section 3 contains some useful computations. In $\S 4$ we consider some general Borsuk-Ulam and Bourgin-Yang theorems together with parametrized versions. Applications, including the Lasry-Magill problem, are given in $\S 5$. Finally $\S 6$ gives a brief preview (without proofs) of how the ideal-valued theory fits into the Ljusternik-Schirelmann method in critical point theory. A worthwhile feature of this approach is the estimate of the ideal Index ${ }^{G} K_{c}$ (see (3) of $\$ 6$ ), where $K_{c}$ is a critical set. Previous estimates of the 'size of $K_{c}$ ' require that the numerical-valued indices be $a$ priori finite (e.g. [4] and [5]) and in the contrary case fail to give any definite information.

\section{Index thoery}

The idea of an ideal-valued cohomological index theory can be formulated quite generally. We will not pursue this generality in great detail and will specialize quickly to the case of paracompact $G$-pairs and $G$-cohomology. But first consider any category $\mathscr{P}$ of topological pairs $(X, A)$ and maps and let $h(X, A)$ denote a multiplicative cohomology theory on $\mathscr{P}$, i.e. $h$ is a contravariant functor into graded algebras over a field $\mathbb{K}$, and $h$ is equipped with long exact sequences, excision, the homotopy property, and a unit in $h(X)$ (where $h(X, \varnothing)=h(X)$ ), when $X \neq \varnothing$. Let $B$ be a fixed space in $\mathscr{P}$ and $p:(X, A) \rightarrow(B, B)$ a map in $\mathscr{P}$, called a pair over $B$. We will often write $p:(X, A) \rightarrow B$ for short. Then we have $h(p): h(B) \rightarrow h(X)$ and the given multiplication $h(X, A) \otimes h(X) \rightarrow h(X, A)$, which together give $h(X, A)$, the structure of a right module over the ring $h(B)$.

Definition 2.1. Index ${ }_{B}(X, A)=$ Annih $h(X, A) \subset h(B)$, i.e. Index In $_{B}(X, A)$ is the ideal in the ring $h(B)$ which annihilates $h(X, A)$.

Remark 2.2. The corresponding numerical index $\left|\operatorname{Index}_{B}(X, A)\right|=$ $\operatorname{dim}_{R}\left[h(X, A) / \operatorname{Index}_{B}(X, A)\right]$ was studied for Borel cohomology in [1] and [5].

Our main concern in this paper will be with the absolute case in definition 2.1 , i.e. $A=\varnothing$. In this case we observe first that

$$
\text { Index }_{B} X=\operatorname{ker} h(p), \quad p: X \rightarrow B .
$$

Next, Index ${ }_{B} X$ possesses the following simple properties.

(a) (monotone) If $f: X_{1} \rightarrow X_{2}$ is a map over $B$, then

$$
\text { Index }_{B} X_{1} \supset \text { Index }_{B} X_{2} \text {. }
$$

(b) (additive) If $\left\{X_{1} \cup X_{2} ; X_{1}, X_{2}\right\}$ is an excisive pair in $\mathscr{P}$ and $p: X_{1} \cup X_{2} \rightarrow B$, then

$$
\text { Index }_{B} X_{1} \cdot \text { Index }_{B} X_{2} \subset \operatorname{Index}_{B}\left(X_{1} \cup X_{2}\right)
$$

or, in terms of ideal quotients [1],

$$
\text { Index }_{B} X_{2} \subset\left(\operatorname{Index}_{B}\left(X_{1} \cup X_{2}\right): \operatorname{Index}_{B} X_{1}\right) \text {. }
$$

The third basic property is that of continuity, which requires a continuous cohomology theory in the following sense. Suppose $(X, A)$ in $\mathscr{P}$, where $A$ is closed 
in $X, X$ is normal, and closed subsets of $X$ belong to $\mathscr{P}$. If $\mathcal{N}$ is the family of neighbourhoods $\mathcal{N}$ of $A, \mathcal{N} \in \mathscr{P}$ directed by inclusion, we assume $\underline{\lim } h(N)=h(A)$. If $\Lambda=h(\mathrm{pt})$, then $h(B)$ is a $\Lambda$-module and we further assume that $X$ is a space over $B$ and $h(B)$ is a Noetherian $\Lambda$-module.

(c) (continuity) There is an open set $U$ such that $A \subset U \subset \vec{U} \subset X$ and Index $_{B} \bar{U}=\operatorname{Index}_{B} A$.

Remark. For proofs of (a), (b) and (c) use the same techniques as the results in [4] for the associated numerical-valued index theory. There are also corresponding results for the relative index $\operatorname{Index}_{B}(X, A)$ as well as for another useful index theory denoted by $\delta$-Index ${ }_{B}(X, A)$, which is defined as the annihilator of image $\delta: h(A) \rightarrow$ $h(X, A)$, where $\delta$ is the coboundary operator in the cohomology theory $h$.

The index theory primarily of interest to us here is a special case of the following. $G$ will be a compact Lie group and $\mathscr{P}$ the category of paracompact $G$-pairs $(X, A)$, i.e. $X$ is a paracompact (Hausdorff) $G$-space and $A$ is a closed $G$-subset. If $B$ is a fixed paracompact $G$-space and $p:(X, A) \rightarrow(B, B)$ is a $G$-map, we have a $G$-pair over $B$, also written simply as $p:(X, A) \rightarrow B$. The cohomology theory $h(X, A)=$ $H_{G}^{*}(X, A)$ is Borel cohomology based on Alexander-Spanier cohomology $H^{*}$. If $E=E G$, the total space of the universal $G$-bundle $E G \rightarrow B G$, then $H_{G}^{*}(X, A)=$ $H^{*}\left(E \times{ }_{G} X, E \times{ }_{G} A\right)$ with coefficients in some field $K$. The corresponding index theory will be denoted by $\operatorname{Index}_{B}^{G}(X, A)$. Thus Index ${ }_{B}^{G}(X, A)$ is an ideal in the ring $H_{G}^{*}(B)$. Note that $H_{G}^{*}(X, A)$ is also an ideal over $\Lambda=H^{*}(B G)$. When $B$ is a point, $H_{G}^{*}(\mathrm{pt})=H^{*}\left(E \times_{G} \mathrm{pt}\right)=H^{*}(B G)$. In this case $\operatorname{Index}_{B}^{G}(X, A)$ is denoted simply by Index ${ }^{G}(X, A)$ and is an ideal in $H^{*}(B G)$.

The absolute case Index ${ }^{G} X$ will occupy most of our attention and in this case Index ${ }^{G} X=\operatorname{ker}\left(H^{*}(B G) \rightarrow H_{G}^{*}(X)\right)$, where $E \times_{G} X \rightarrow B G$ is induced by the projection $E \times X \rightarrow E$.

\section{Computations}

Our first computation is a product formula. We suppose $p_{1}: X_{1} \rightarrow B_{1}, p_{2}: X_{2} \rightarrow B_{2}$ are $G_{1}$ - and $G_{2}$-spaces over $B_{1}$ and $B_{2}$, and let $p=p_{1} \times p_{2}: X_{1} \times X_{2} \rightarrow B_{1} \times B_{2}$ denote the $G$-space over $B_{1} \times B_{2}$, where $G=G_{1} \times G_{2}$. We do not assume $B_{1}$ and $B_{2}$ are trivial $G_{1}$ - and $G_{2}$-spaces. Then $p$ induces

$$
p_{G}:\left(E_{1} \times E_{2}\right) \times_{G}\left(X_{1} \times X_{2}\right) \rightarrow\left(E_{1} \times E_{2}\right) \times_{G}\left(B_{1} \times B_{2}\right),
$$

which can be identified with

$$
p_{1 G_{1}} \times p_{2 G_{2}}:\left(E_{1} \times_{G_{1}} X_{1}\right) \times\left(E_{2} \times_{G_{2}} X_{2}\right) \rightarrow\left(E_{1} \times_{G_{1}} B_{1}\right) \times\left(E_{2} \times_{G_{2}} B_{2}\right),
$$

and hence $p_{G}^{*}$ may be identified with (coefficients in the field $K$ )

$$
p_{i G_{1}}^{*} \otimes p_{2 G_{2}}^{*}: H_{G_{1}}^{*}\left(B_{1}\right) \otimes H_{G_{2}}^{*}\left(B_{2}\right) \rightarrow H_{G_{1}}^{*}\left(X_{1}\right) \otimes H_{G_{2}}^{*}\left(X_{2}\right)
$$

in the presence of appropriate finiteness conditions, e.g. where $H_{G_{1}}^{*}\left(B_{1}\right)$ and $H_{G_{1}}^{*}\left(X_{1}\right)$ are of finite type (over $K$ ). If we let $\mu_{1}=\operatorname{Index}_{B_{1}^{1}}^{G_{1}} X_{1}, \mu_{2}=\operatorname{Index}_{B_{2}}^{G} X_{2}$ and $h=$ Index $B_{1} \times B_{2}\left(X_{1} \times X_{2}\right)$, then we have the following:

Proposition 3.1. $\mu=h_{1} \otimes H_{G_{2}}^{*}\left(B_{2}\right)+H_{G_{1}}^{*}\left(B_{1}\right) \otimes h_{2}$.

Proof. This is given by a simple vector space argument over $\mathbb{K}$. 
CoRollary 3.2. If $H^{*}\left(B G_{1}\right)=\mathbb{K}\left[x_{1}, \ldots, x_{k}\right], \quad H^{*}\left(B G_{2}\right)=\mathbb{K}\left[y_{1}, \ldots, y_{i}\right], \quad h_{1}=$ $\left\{f_{1}, \ldots, f_{m}\right\}$ and $k_{2}=\left\{g_{1}, \ldots, g_{n}\right\}$, where $f_{i}$ and $g_{i}$ are polynomials in the $x$ and $y$ respectively, then

$$
h=\left\{f_{1}, \ldots, f_{m}, g_{1}, \ldots, g_{n}\right\},
$$

the ideal generated by the polynomials $f_{i}, g_{j}$.

Example 3.3. Let $S^{p}$ denote the $p$-sphere with a free $\mathbb{Z}_{2}$-action. Then let $\mathbb{K}=\mathbb{Z}_{2}$ so that in this case $H^{*}(B G)=\mathbb{Z}_{2}[t], \operatorname{dim} t=1$. Then Index ${ }^{Z_{2}} S^{p}=\left\{t^{p+1}\right\}$, the ideal generated by $t^{p+1}$. If $T(k)=\mathbb{Z}_{2} \times \cdots \times \mathbb{Z}_{2}$ ( $k$ times), then $T(k)$ acts freely on $S^{n_{1}} \times \cdots \times S^{n_{k}}$ and

$$
\operatorname{Index}^{T(k)} S^{n_{1}} \times \cdots \times S^{n_{k}}=\left\{t_{1}^{n_{1}+1}, t_{2}^{n_{2}+1}, \ldots, t_{k}^{n_{k}+1}\right\}
$$

in the polynomial ring $\mathbb{Z}_{2}\left[t_{1}, \ldots, t_{k}\right]$.

COROLlary 3.4. if we set $X_{2}=B_{2}=\mathrm{pt}$ in proposition 3.1, we obtain

$$
\text { Index } X_{B_{1}}^{G_{1} \times G_{2}} X_{1}=\left(\operatorname{Index} B_{B_{1}}^{G_{1}} X_{1}\right) \otimes H^{*}\left(B G_{2}\right) \text {, }
$$

where $G_{1} \times G_{2}$ acts on $X_{1}$ and $B_{1}$ by $\left(g_{1}, g_{2}\right) x=g_{1} x$.

Proposition 3.5. Let $X_{1}$ and $X_{2}$ denote $G_{1}$-and $G_{2}$-spaces (over $\mathrm{pt}$ ). Then the join $X_{1} * X_{2}$ is $a\left(G_{1} \times G_{2}\right)$-space via the action $\left(g_{1}, g_{2}\right)\left(x_{1}, t, x_{2}\right)=\left(g_{1} x_{1}, t, g_{2} x_{2}\right)$ and Index ${ }^{G_{1} \times G_{2}}\left(X_{1} * X_{2}\right) \subset\left[\left(\operatorname{Index}^{G_{1}} X_{1}\right) \otimes H^{*}\left(B G_{2}\right)\right] \cap\left[\left(H^{*}\left(B G_{1}\right) \otimes \operatorname{Index}^{G_{2}} X_{2}\right)\right]$, where $\cap$ represents the intersection of ideals.

Proof. $X_{1}$ and $X_{2}$ appear in $X_{1} * X_{2}$ as $\left(G_{1} \times G_{2}\right)$-subspaces, so that

$$
\text { Index }{ }^{G_{1} \times G_{2}} X_{i} \supset \text { Index }{ }^{G_{1} \times G_{2}} X_{1} * X_{2}, \quad i=1,2 .
$$

An application of corollary 3.4 completes the proof.

Proposition 3.6. Let $X_{1}$ and $X_{2}$ be as in proposition 3.5. Then

$$
\text { Index }{ }^{G_{1} \times G_{2}}\left(X_{1} * X_{2}\right) \supset\left[\left(\operatorname{Index}^{G_{1}} X_{1}\right) \otimes H^{*}\left(B G_{2}\right)\right] \cdot\left[H^{*}\left(B G_{1}\right) \otimes \operatorname{Index}^{G_{2}} X_{2}\right],
$$

where represents the product of ideals.

Proof. Observe that $X_{1} * X_{2}=Y_{1} \cup Y_{2}$, where $Y_{i}$ is a closed $\left(G_{1} \times G_{2}\right)$-subspace equivalent to $X_{i}$ and the additivity property applies.

Let $h_{1}=$ Index $^{G_{1}} X_{1}$ and $h_{2}=$ Index $^{G_{2}} X_{2}, \Lambda_{1}=H^{*}\left(B G_{1}\right), \Lambda_{2}=H^{*}\left(B G_{2}\right)$. If we identify $h_{1}$ with $h_{1} \otimes 1$ and $h_{2}$ with $1 \otimes h_{2}$, then $h_{1} \cdot h_{2}=\left(h_{1} \otimes \Lambda_{2}\right) \cdot\left(\Lambda_{1} \otimes h_{2}\right)$ and the conclusion of proposition 3.6 reads

$$
\text { Index }{ }^{G_{1} \times G_{2}}\left(X_{1} * X_{2}\right) \supset\left(\text { Index }{ }^{G_{1}} X_{1}\right) \cdot\left(\text { Index }{ }^{G_{2}} X_{2}\right) \text {. }
$$

Proposition 3.7. When $\left(\mu_{1} \otimes \Lambda_{2}\right) \cap\left(\Lambda_{1} \otimes h_{2}\right)=h_{1} \cdot h_{2}$, we have

$$
\text { Index }{ }^{G_{1} \times G_{2}}\left(X_{1} * X_{2}\right)=\left(\operatorname{Index}^{G_{1}} X_{1}\right) \cdot\left(\text { Index }{ }^{G_{2}} X_{2}\right) \text {. }
$$

As special cases, let $G=\mathbb{Z}_{2}, S^{1}$, or $S U(2), \mathbb{K}_{2}=\mathbb{Z}_{2}$ or $\mathbb{Q}$ so that $H^{*}(B G)=\mathbb{K}[t]$, the polynomial ring on a single indeterminate $t$. Let $d=\operatorname{dim} t$ so that $d=1,2$ or 4 and suppose $G$ acts freely on $S^{p}$ and $S^{q}$, where $p=d k-1$ and $q=d l-1$. Then

$$
\text { Index }{ }^{G} S^{p}=\left\{t_{1}^{k}\right\}, \quad \text { Index }^{G} S^{q}=\left\{t_{2}^{l}\right\}
$$

and

$$
H^{*}(B G) \otimes H^{*}(B G)=\mathbb{K}\left[t_{1}, t_{2}\right] .
$$


Proposition 3.8

$$
\text { Index }{ }^{G \times G}\left(S^{p} * S^{q}\right)=\left\{t_{1}^{k} t_{2}^{l}\right\} \subset \mathbb{K}\left[t_{1}, t_{2}\right] .
$$

Proof. Proposition 3.7 applies.

If we represent $S^{N}$ as $S^{n_{1}} * S^{n_{2}} * \cdots * S^{n_{k}}$ and let $\mathbb{Z}_{2}$ act freely on $S^{n_{i}}$ so that $T(k)=\mathbb{Z}_{2} \times \cdots \times \mathbb{Z}_{2}(k$ times $)$ operates on $S^{N}$ component-wise, then we have the following:

Corollary 3.9

$$
\text { Index }^{T(k)}\left(S^{n_{1}} * S^{n_{2}} * \cdots * S^{n_{k}}\right)=\left\{t_{1}^{n_{1}+1} t_{2}^{n_{2}+1} \cdots t_{k}^{n_{k}+1}\right\},
$$

the ideal generated by the single monomial $t_{1}^{n_{1}+1} t_{2}^{n_{2}+1} \cdots t_{k}^{n_{k}+1}$. In particular

$$
\text { Index } \left.^{T(k)} \overline{\left(S^{q-1}\right.} * S^{q-1} * \cdots * S^{q-1}\right)=\left(t_{1} t_{2} \cdots t_{k}\right)^{q} \text {. }
$$

Proof. This is left to the reader, using proposition 3.7 .

Remark 3.10. If $G=S^{1}$ or $S U(2)$ and we let $T(k)$ denote $S^{1} \times \cdots \times S^{1}$ or $S U(2) \times$ $\cdots \times S U(2)$ ( $k$ times) and $S^{1}$ and $S U(2)$ operate freely on the $S^{n_{i}}$, the results corresponding to example 3.3 and corollary 3.9 obtain with the necessary arithmetic modifications.

Observation 3.11. The dimension of the sphere in corollary 3.9 is $n+k-1$, where $n=\sum n_{i}$. In the spectral sequence of the fibration $S^{n+k-1} \rightarrow E \times_{T(k)} S^{n+k-1} \rightarrow B T(k)$ the differential operator $d_{n+k}$ must send the generator of $S^{n+k-1}$ to the monomial $t_{1}^{n_{1}+1} t_{2}^{n_{2}+1} \cdots t_{k^{k}}^{n_{k}+1}$ and this forces $H^{*}(B T(k)) \rightarrow H_{T(k)}^{*}\left(S^{n+k-1}\right)$ to be surjective. This fact will be used later.

Now we consider $G$-spaces $X_{1}$ and $X_{2}$ (over points) and let $G$ act on $X \times Y$ and $X * Y$ diagonally, i.e. $g(x, y)=(g x, g y), g(x, t, y)=(g x, t, g y)$.

Proposition 3.12

$$
\text { Index }^{G}\left(X_{1} \times X_{2}\right) \supset\left(\text { Index }^{G} X_{1}\right) \cap\left(\operatorname{Index}^{G} X_{2}\right) .
$$

Proof. Consider the projection $X_{1} \times X_{2} \rightarrow X_{i}, i=1,2$. The monotone property implies Index $^{G}\left(X_{1} \times X_{2}\right) \supset \operatorname{Index}^{G} X_{i}$.

Proposition 3.13

$$
\text { Index }{ }^{G} X_{1} * X_{2} \supset\left(\text { Index }^{G} X_{1}\right) \cdot\left(\text { Index }{ }^{G} X_{2}\right) \text {. }
$$

Proof. This is left to the reader.

Remark 3.14. In general,

$$
\text { Index }{ }^{G}\left(X_{1} * X_{2}\right) \neq\left(\operatorname{Index}^{G} X_{1}\right) \cdot\left(\operatorname{Index}^{G} X_{2}\right) \text {. }
$$

As an example let $G$ act trivially on $X_{1}$. Then

$$
\text { Index }{ }^{G} X_{1}=H^{*}(B G)=\operatorname{Index}^{G} X_{1} * X_{2} \supset \text { Index } X_{1} \cdot \operatorname{Index}^{G} X_{2}=\operatorname{Index}^{G} X_{2} \text {. }
$$

Now choose any $X_{2}$ such that $\operatorname{Index}^{G} X_{2}$ is a proper ideal.

Remark 3.15. We note here that if $X$ is a free $G$-space, then Index ${ }^{G} X$ coincides with $\operatorname{ker} H^{*}(B G) \rightarrow H^{*}(X / G)$, where $X / G \rightarrow B G$ is the classifying map [2].

We now consider a basic computation which is important to an application which we give later on. $V_{n, k}$ will denote the space of orthonormal $k$-frames in $\mathbb{R}^{n}$ and $O(k)$ 
will denote the orthogonal group on $\mathbb{R}^{k}$. Then $O(k)$ operates freely on $V_{n, k}$ by the usual action $g v, g \in O(k)$ and $v$ a column vector representing the $k$-frame. We restrict this action to the subgroup $T(k)$ of diagonal matrices with entries \pm 1 and compute Index $T^{(k)} V_{n, k}$. The computation will be based on the fibration

$$
V_{n-k+1,1} \stackrel{i}{\longrightarrow} V_{n, k} \stackrel{\pi}{\longrightarrow} V_{n, k-1},
$$

where $\pi$ is the projection on the first $k-1$ coordinates. Consider the sequence

$$
\mathbb{Z}_{2} \rightarrow T(k) \rightarrow T(k-1)
$$

where $\mathbb{Z}_{2}$ injects on the last coordinate and $T(k)$ projects on the first $k-1$ coordinates. Dividing out the action of (2) on (1), we obtain a fibration

$$
\mathbb{R} P^{n-k} \stackrel{\tilde{i}}{\longrightarrow} \tilde{V}_{n, k} \stackrel{\bar{\pi}}{\longrightarrow} \tilde{V}_{n, k-1} .
$$

We then have an induced diagram of fibrations

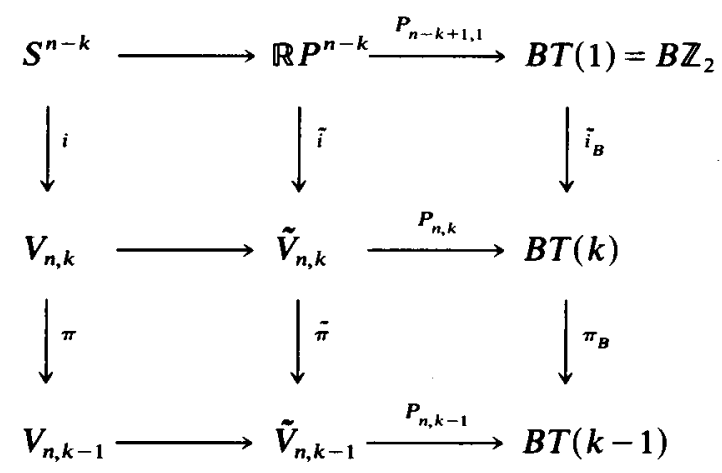

where the $p_{i, j}$ are classifying maps. Recall that our coefficients are $\mathbb{Z}_{2}$, and since $\tilde{i}_{B}^{*}$ and $p_{n-k+1,1}^{*}$ are surjective, $\tilde{i}^{*}: H^{*}\left(\tilde{V}_{n}, k\right) \rightarrow H^{*}\left(\mathbb{R} P^{n-k}\right)$ is surjective and the LerayHirsch theorem [8] applies. If $\theta: H^{*}\left(\mathbb{R} P^{n-k}\right) \rightarrow H^{*}\left(\tilde{V}_{n, k}\right)$ is a right inverse for $\tilde{i}^{*}$ and $u \in H^{1}\left(\mathbb{R} P^{n-k}\right)$ is the generator, we may assume that if $H^{*}(B T(k))=$ $\mathbb{Z}_{2}\left[t_{1}, \ldots, t_{k}\right]$, then $\theta(u)=p_{n, k}^{*}\left(t_{k}\right)$ and, in fact, $\theta\left(u^{i}\right)=p_{n, k}^{*}\left(t_{k}^{i}\right), 1 \leq i \leq n-k$. We then have an isomorphism of $H^{*}\left(\tilde{V}_{n, k-1}\right)$-modules

$$
\varphi_{k}: H^{*}\left(\tilde{V}_{n, k-1}\right) \otimes H^{*}\left(\mathbb{R} P^{n-k}\right) \rightarrow H^{*}\left(\tilde{V}_{n, k}\right)
$$

given by $\varphi_{k}(x \otimes y)=\tilde{\pi}^{*}(x) \cdot \theta(y)$.

Note that $\tilde{i}^{*}\left(\tilde{\pi}^{*}(x) \cdot \theta(y)\right)=0$ if $\operatorname{dim} x>0$ and $\tilde{i}^{*}\left(\tilde{\pi}^{*}(1) \cdot \theta(y)\right)=y$, so that the kernel of $\tilde{i}^{*}$ corresponds precisely $\tilde{H}^{*}\left(\tilde{V}_{n, k}\right) \otimes H^{*}\left(\mathbb{R} P^{n-1}\right)$. Set $\bar{t}_{i}=p_{n, k}\left(t_{i}\right), 1 \leq i \leq k$, and note that if we assume inductively that $p_{n, k-1}^{*}$ is surjective, then $p_{n, k}^{*}$ is surjective and $\tilde{H}^{*}\left(\tilde{V}_{n, k}\right)$ is generated by $\bar{t}_{1}, \ldots, \bar{t}_{k}$. We are now in a position to state our computational result on the index of $V_{n, k}$.

TheOREM 3.16. Index ${ }^{T(k)}=\left\{f_{1}, \ldots, f_{k}\right\} \subset \mathbb{Z}_{2}\left[t_{1}, \ldots, t_{k}\right]$ where $\left\{f_{1}, \ldots, f_{k}\right\}$ is the ideal generated by polynomials $f_{1}, \ldots, f_{k}$. Here $f_{i}$ has the form

$$
f_{i}=t_{i}^{n-i+1}+w_{i, n-1} t_{i}^{n-i}+\cdots+w_{i, 0},
$$

where $w_{i, j}$ are polynomials in $t_{1}, \ldots, t_{i-1}$ and $w_{i, j} t^{j}$ is of degree $n-i+1$. In particular $\left(t_{1} t_{2} \cdots t_{k}\right)^{n-k}$ is not contained in Index ${ }^{T(k)}\left(V_{n, k}\right)$. 
Proof. We proceed by induction on $k$. Consider the element $\bar{t}_{k}^{n-k+1}$. Since $\tilde{i}^{*}\left(\bar{t}_{k}^{n-k+1}\right)=$ $0, \bar{t}_{k}^{n-k+1}$ has the form

Hence

$$
\bar{t}_{k}^{n-k+1}=\bar{w}_{k, n-k} \bar{t}_{k}^{n-k}+\cdots+\bar{w}_{k, 0}
$$

$$
\bar{t}_{k}^{n-k+1}-\bar{w}_{k, n-k} \bar{t}_{k}^{n-k}-\cdots-\bar{w}_{k, 0}
$$

or

$$
f_{k}=t_{k}^{n-k+1}+w_{k, n-k} t_{k}^{n-k}+\cdots+w_{k, 0}
$$

is in the kernel of $p_{n, k}^{*}$, where $p_{n, k}^{*}\left(w_{k, j}\right)=-\bar{w}_{k, j}$. By induction, $\left\{f_{1}, \ldots, f_{k}\right\} \subset \operatorname{ker} p_{n, k}^{*}=$ Index ${ }^{T(k)} V_{n, k}$. Now a simple algebraic argument shows the reverse inclusion. It is also easy to see that $\left(t_{1} t_{2} \cdots t_{k}\right)^{n-k}$ cannot be expressed in terms of $f_{1}, \ldots, f_{k}$. The latter can also be seen inductively as follows. Write

$$
\left(t_{1} t_{2} \cdots t_{k}\right)^{n-k}=\left(t_{1} t_{2} \cdots t_{k-1}\right)^{n-k} t_{k}^{n-k}
$$

and since $\left(\bar{t}_{1} \bar{t}_{2} \cdots \bar{t}_{k-1}\right)^{n-k+1} \neq 0,\left(\bar{t}_{1} \bar{t}_{2} \cdots \bar{t}_{k-1}\right)^{n-k} \cdot \bar{t}_{k}^{n-k} \neq 0$ using the isomorphism $\varphi_{k}$. Thus $\left(t_{1} t_{2} \cdots t_{k}\right)^{n-k}$ is not in the kernel of $p_{n, k}^{*}=\operatorname{Index}^{T(k)} V_{n, k}$.

4. Borsuk-Ulam-Bourgin-Yang (BUBY) theorems and the parameter principle We first formulate and prove a general Bourgin-Yang theorem as follows. Let $V$ and $V^{\prime}$ denote $G$-spaces and $Z^{\prime}$ a closed $G$-set in $V^{\prime}$. We assume through this section that $\Lambda=H^{*}(B G)$ is Noetherian.

THEOREM 4.1, Let $f: V \rightarrow V^{\prime}$ be $a$ G-map and $Z=f^{-1}\left(Z^{\prime}\right)$. Then $Z$ is $a$ G-set and

$$
\text { Index }{ }^{G} Z \subset\left(\text { Index }^{G} V: \text { Index }{ }^{G}\left(V^{\prime}-Z^{\prime}\right)\right) \text {. }
$$

Proof. The proof uses the three basic properties as follows. Using the continuity property, choose a $G$-neighbourhood $U$ of $Z$ such that $Z \subset U \subset \bar{U}$ and Index ${ }^{G} \bar{U}=\operatorname{Index}^{G} Z$. Then additivity implies that

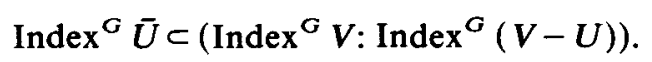

Finally, the monotone property implies that

$$
\operatorname{Index}^{G}(V-U) \supset \operatorname{Index}^{G}\left(V^{\prime}-Z^{\prime}\right)
$$

and hence

$$
\text { Index }{ }^{G} Z \subset\left(\text { Index }^{G} V: \text { Index }^{G}\left(V^{\prime}-Z^{\prime}\right)\right) \text {. }
$$

A special case of the above theorem which is also an immediate consequence of the monotone property is the following:

Proposition 4.2. Let $f: V \rightarrow V^{\prime}$ be $a$ G-map and $Z=f^{-1}\left(Z^{\prime}\right)$. Then, if Index ${ }^{G} V \not \supset$ Index ${ }^{G}\left(V^{\prime}-Z^{\prime}\right), Z \neq \varnothing$.

We prove next a parametrized version of the above theorem. We are indebted to A. Dold for a suggestion which led to this general formulation. Our parameter space $P$ will be compact, connected, smooth manifold (possibly with boundary), orientable over our coefficient field $\mathbb{K}$. 
Proposition 4.3 (parameter principle). Let $P$ denote a parameter space and $p_{0} \epsilon$ $P-\partial P$. Let $V$ denote a compact, connected, smooth manifold, orientable over $\mathbb{K}$. $f:(P, \partial P) \times V \rightarrow(P, \partial P)$ is a given $G$-map such that $f_{y}:(P, \partial P) \rightarrow(P, \partial P)$ has non-zero degree, where $f_{y}(x)=f(x, y), x \in P, y \in V$. Then $W=f^{-1}\left(p_{0}\right)$ is a G-set and Index ${ }^{G} W=\operatorname{Index}^{G} V$.

Proof. (a) We first prove the proposition in the case when $V$ is a free $G$-space. If $\tilde{V}$ denotes the orbit space $V / G$, then $f$ induces

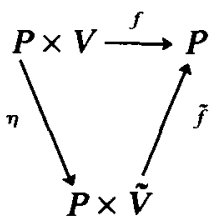

and $\eta(W)=\tilde{W}=\tilde{f}^{-1}\left(p_{0}\right)$. Choose a $G$-neighbourhood $U$ of $W$ such that $W \subset U \subset \bar{U}$ and Index ${ }^{G} \bar{U}=\operatorname{Index}^{G} W$. Let $\tilde{g}$ denote an approximation of $\tilde{f}$ so that $\tilde{g} \pitchfork p_{0}$, i.e. $p_{0}$ is a regular value for $\tilde{g}$ and $\tilde{N}=\tilde{g}^{-1}\left(p_{0}\right) \subset \tilde{U}$. Then $\tilde{N}$ is a submanifold with $\operatorname{dim} \tilde{N}=\operatorname{dim} \tilde{V}$. Let $N=\eta^{-1}(\tilde{N})$ and $g=\tilde{g} \eta$. Then we have a diagram

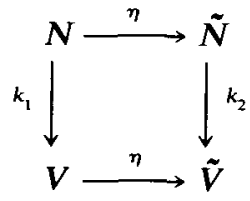

where $k_{1}$ and $k_{2}$ are the usual projections. Note that $\operatorname{deg} f_{y}=\operatorname{deg} g_{y}, y \in V$. Also, it is easy to check, because $k_{1}(u, v)=y$ if and only if $v=y$ and $g(u, y)=p_{0}$, that $\operatorname{deg} k_{1}=\operatorname{deg} k_{2}=\operatorname{deg} f_{y} \neq 0$. We may apply the 'umkehr homomorphism' (Hopf transfer [3]) to the map $k_{2}$ to conclude that $k_{2}^{*}: H^{*}(\tilde{V}) \rightarrow H^{*}(\tilde{N})$ is injective. Since the action is free, we have that

$$
k_{1 G}^{*}: H_{G}^{*}(V) \rightarrow H_{G}^{*}(N)
$$

is also injective and hence Index ${ }^{G} N=\operatorname{Index}^{G} V$. But $N \subset \bar{U}$ implies Index ${ }^{G} N \supset$ Index ${ }^{G} \bar{U}=\operatorname{Index}^{G} W$. Hence Index ${ }^{G} V \supset \operatorname{Index}^{G} W$. On the other hand the projection map $W \rightarrow V$ is a $G$-map and hence Index ${ }^{G} W \supset \operatorname{index}^{G} V$. Thus in the free case Index ${ }^{G} W=\operatorname{Index}^{G} V$.

(b) To prove the general case, we reduce to the free case as follows. Recall that $E=E G$ is the limit of compact, smooth, free $G$-manifolds $E^{1} \subset E^{2} \subset \cdots \subset E^{m} \subset \cdots$, with the property that the inclusion map $i_{m}$ induces isomorphisms

$$
H^{q}\left(E \times_{G} X\right) \rightarrow H^{q}\left(E^{m} \times_{G} X\right)
$$

for $q<m, X$ a paracompact $G$-space [6]. Thus

$$
\bigcap_{m} \operatorname{Index}^{G}\left(E^{m} \times X\right)=\operatorname{Index}^{G} X .
$$

Now, in the general case, for each value of $m$ we may apply (a) to the map

$$
\bar{f}: P \times\left(E^{m} \times V\right) \rightarrow P,
$$


where $\bar{f}(x, e, y)=f(x, y)$, to obtain

$$
\text { Index }{ }^{G}\left(E^{m} \times V\right)=\operatorname{Index}^{G}\left(E^{m} \times W\right)
$$

for each $m$, and hence

$$
\text { Index }{ }^{G} V=\operatorname{Index}^{G} W
$$

We now have the parametrized version of theorem 4.1 .

TheOREM 4.4. Let $P$ denote a parameter space (as described above) and $V$ a compact, connected manifold which is orientable over $\mathbb{K}$. Let $V^{\prime}$ denote a $G$-space and

$$
\varphi=(f, g):(P, \partial P) \times V \rightarrow(P, \partial P) \times V^{\prime}
$$

a G-map such that $f_{y}:(P, \partial P) \rightarrow(P, \partial P), y \in V$, has non-zero degree over $\mathbb{K}$. If $Z^{\prime} \subset V^{\prime}$ is a closed subset of $V^{\prime}, p_{0} \in P-\partial P$, and $Z\left(p_{0}\right)=\varphi^{-1}\left(p_{0} \times Z^{\prime}\right)$, then

$$
\text { Index }{ }^{G} Z\left(p_{0}\right) \subset\left(\text { Index }^{G} V: \text { Index }^{G}\left(V^{\prime}-Z^{\prime}\right)\right) \text {. }
$$

Proof. Let $W=f^{-1}\left(p_{0}\right)$. Then by the parameter principle Index ${ }^{G} W=\operatorname{Index}^{G} V$. Consider

$$
g_{0}=g \mid W: W \rightarrow V^{\prime}
$$

and note that $g_{0}^{-1}\left(Z^{\prime}\right)=Z\left(p_{0}\right)$. Applying theorem 4.1 gives

$$
\text { Index }{ }^{G} Z\left(p_{0}\right) \subset\left(\operatorname{Index}^{G} V: \operatorname{Index}^{G}\left(V^{\prime}-Z^{\prime}\right)\right) \text {. }
$$

Remark. As pointed out by the referee, proposition 4.3 , and consequently theorem 4.4 , can be extended to the case where $V$ is a compact $G$-subset of some $\mathbb{R}^{N}$, but this extension is not required here.

Remark 4.5. We note that the right-hand side of the inclusion

$$
\text { Index }{ }^{G} Z\left(p_{0}\right) \subset\left(\text { Index }^{G} V: \text { Index }^{G}\left(V^{\prime}-Z^{\prime}\right)\right)
$$

is independent of $P$ and $p_{0}$. Thus, for appropriate $V$, the parametrized version follows immediately after one has established the result for the single space $V$.

\section{Applications}

We will give two applications, both involving the Euclidean space $\left(\mathbb{R}^{k}\right)^{n-k} . O(k)$ acts in the standard way on $\mathbb{R}^{k}$ and hence $O(k)$ acts on $\mathbb{R}^{k} \times \cdots \times \mathbb{R}^{k}(n-k$ times) in a coordinate-wise manner. Let $T(k) \subset O(k)$ denote the subgroup of diagonal matrices $\operatorname{diag}\left[\varepsilon_{1}, \ldots, \varepsilon_{k}\right]$, where $\varepsilon_{i}= \pm 1$. Thus $T(k)=\mathbb{Z}_{2} \times \cdots \times \mathbb{Z}_{2}(k$ times) and $\left(\mathbb{R}^{k}\right)^{n-k}$ is a $T(k)$-space. We may also represent $\left(\mathbb{R}^{k}\right)^{n-k}$ as $\left(\mathbb{R}^{n-k}\right)^{k}$, where $\mathbb{Z}_{2}$ acts freely on each $\mathbb{R}^{n-k}-0$. Thus, as a $T(k)$-space $\left(\mathbb{R}^{k}\right)^{n-k}-0$ is the same $T(k)$ homotopy type as the $T(k)$-space,

$$
S^{k(n-k)-1}=S^{n-k-1} * \cdots * S^{n-k-1} \quad(k \text { times }) .
$$

Applying corollary 3.9 , we have

$$
\text { Index }^{T(k)}\left(\left(\mathbb{R}^{k}\right)^{n-k}-0\right)=\left\{\left(t_{1} t_{2} \cdots t_{k}\right)^{n-k}\right\} \subset \mathbb{Z}_{2}\left[t_{1}, \ldots, t_{k}\right] .
$$

We recall next the $T(k)$-space $S^{n-1} \times S^{n-2} \times \cdots \times S^{n-k}$ of example 3.3, i.e. each $\mathbb{Z}_{2}$ factor of $T(k)$ acts freely on the corresponding sphere. Then

$$
\text { Index }{ }^{T(k)} S^{n-1} \times \cdots \times S^{n-k}=\left\{t_{1}^{n}, t_{2}^{n-1}, \ldots, t_{k}^{n-k+1}\right\} \text {. }
$$


THEOREM 5.1. Let $f: S^{n-1} \times \cdots \times S^{n-k} \rightarrow\left(\mathbb{R}^{k}\right)^{n-k}$ denote a $T(k)-$ map. Then $f$ has an orbit of zeros.

Proof. Since $\left(t_{1} t_{2} \cdots t_{k}\right)^{n-k} \notin\left\{t_{1}^{n}, t_{2}^{n-1}, \ldots, t_{k}^{n-k+1}\right\}$, corollary 4.2 applies and the proof is complete.

The Bourgin-Yang theorem 4.1 provides more information. Let $Z=f^{-1}(0)$, where $f$ is as in theorem 5.1. Then

$$
\text { Index }{ }^{T(k)} Z \subset\left(\left\{t_{1}^{n}, \ldots, t_{k}^{n-k+1}\right\}:\left\{\left(t_{1} t_{2} \cdots t_{k}\right)^{n-k}\right\}\right) .
$$

It is easy to see that the monomial

$$
\mu=t_{1}^{k-1} t_{2}^{k-2} \cdots t_{k}^{0} \notin \text { Index }^{T(k)} Z
$$

or alternatively $\mu$ is not in the kernel $\left(\mathbb{Z}_{2}\right.$-coefficients $)$ of

$$
H_{T(k)}^{*}(Z) \leftarrow H^{*}(B T(k)),
$$

the homomorphism which defines Index ${ }^{T(k)} Z$. Thus the cup length of $H_{T(k)}^{*}(Z)$ is at least $k(k-1) / 2$. Since $Z$ is a free $\mathbb{Z}_{2}$-space, $H_{T(k)}^{*}(Z)=H^{*}(\tilde{Z})$, where $\tilde{Z}=$ $Z / T(k)$.

THEOREM 5.2. Let $f: S^{n-1} \times \cdots \times S^{n-k} \rightarrow\left(\mathbb{R}^{k}\right)^{n-k}$ denote $a T(k)$-map and $Z=f^{-1}(0)$. Then, if $\tilde{Z}=Z / T(k)$, the cup length of $\tilde{Z}$ is at least $k(k-1) / 2$ and the category of $\tilde{Z}$ is at least $k(k-1) / 2+1$.

Now, as noted in $\S 4$, we may by the parameter principle extend theorem 5.2 to the corresponding parametrized version as follows.

Theorem 5.3. Let $P$ denote a parameter space (see $\$ 4)$ and

$$
\varphi:(P, \partial P) \times S^{n-1} \times \cdots \times S^{n-k} \rightarrow(P, \partial P) \times\left(\mathbb{R}^{k}\right)^{n-k}
$$

a $T(k)$-map. Take $p_{0} \in P-\partial P$ and let $W=\varphi^{-1}\left(p_{0}, 0\right)$. If $\varphi=(f, g)$ and $f_{y}:(P, \partial P) \rightarrow$ $(P, \partial P)$ has non-zero degree (in $\left.\mathbb{Z}_{2}\right)$, where $f_{y}(x)=f(x, y), x \in P, y \in S^{n-1} \times \cdots \times S^{n-k}$, then

$$
\text { category } \tilde{W} \geq \frac{k(k-1)}{2}+1
$$

where $\tilde{W}=W / T(k)$.

Our next example involves a problem of Lasry and Magill (see [7] for an alternative solution). Let $V_{n, k}$ denote orthonormal $k$-frames in $\mathbb{R}^{n}$, which is an $O(k)$-space as in $\S 3$. The Lasry-Magill problem may be formulated as follows. Let $D^{l}$ denote an $l$-cell on which $O(k)$ acts trivially. Let

$$
\varphi: D^{l} \times V_{n, k} \rightarrow D^{\prime} \times\left(\mathbb{R}^{k}\right)^{n-k}
$$

denote an $O(k)$-map with the following property. If $\varphi=(f, g)$, where $f: D^{l} \times V_{n, k} \rightarrow$ $D^{\prime}$, then $f \mid \partial d^{l} \times V_{n, k}$ is just a projection on the first factor.

Lasry-Magill problem. Show that there exists an $\left(x_{0}, y_{0}\right) \in D^{l} \times V_{n, k}$ such that $\varphi\left(x_{0}, y_{0}\right)=(0,0)$.

Note that $f_{y}:\left(D^{\prime}, \partial D^{\prime}\right) \rightarrow\left(D^{\prime}, \partial D^{\prime}\right)$, where $f_{y}(x)=f(x, y)$, is the identity on $\partial D^{\prime}$, so that $\operatorname{deg} f_{y}=1$. The reader will recognize then that the Lasry-Magill problem is just the parametrized version of the following. 
THEOREM 5.4. Let $f: V_{n, k} \rightarrow\left(\mathbb{R}^{k}\right)^{n-k}$ denote an $O(k)$-map and $Z=f^{-1}(0)$. Then $f$ is also a $T(k)$-map and

$$
\text { Index }{ }^{T(k)} Z \subset\left(\operatorname{Index}^{T(k)} V_{n, k}: \operatorname{Index}{ }^{T(k)}\left(\left(\mathbb{R}^{k}\right)^{n-k}-0\right)\right) .
$$

Since Index ${ }^{T(k)} V_{n, k} \not \supset$ Index ${ }^{T(k)}\left(\left(\mathbb{R}^{k}\right)^{n-k}-0\right), Z \neq \varnothing$.

Proof. Applying theorem 3.16, $\left(t_{1} \cdots t_{k}\right)^{n-k}$ is not in Index ${ }^{T(k)} V_{n, k}$.

Theorem 5.4 may be strengthened as follows. Recall that in theorem 3.16 Index ${ }^{T(k)} V_{n, k}$ is generated by polynomials

$$
\begin{aligned}
& f_{1}=t_{1}^{n}, \\
& f_{2}=t_{2}^{n-1}+w_{2, n-2} t_{2}^{n-2}+\cdots+w_{2,0}, \\
& \vdots \\
& f_{k}=t_{k}^{n-k+1}+w_{k, n-k} t_{n}^{n-k}+\cdots+w_{k, 0},
\end{aligned}
$$

and the monomial $\mu=t_{1}^{k-1} t_{2}^{k-2} \cdots t_{k}^{0}$ is not in the ideal quotient

$$
\left(\left\{f_{1}, \ldots, f_{k}\right\}:\left\{\left(t_{1} \cdots t_{k}\right)^{n-k}\right\}\right) \text {. }
$$

Thus $\mu \notin \operatorname{Index}^{T(k)} Z$.

THEOREM 5.5. Let $f: V_{n, k} \rightarrow\left(\mathbb{R}^{k}\right)^{n-k}$ denote a $T(k)$-map and $Z=f^{-1}(0)$. Then

$$
\text { category } \tilde{Z} \geq \frac{k(k-1)}{2}+1 \text {, }
$$

where $\tilde{Z}=Z / T(k)$, the orbit space of $Z$.

Using the parameter principle, we have the following parametrized version.

THEOREM 5.6. Let $P$ denote a parameter space and

$$
\varphi:(P, \partial P) \times V_{n, k} \rightarrow(P, \partial P) \times\left(\mathbb{R}^{k}\right)^{n-k}
$$

a $T(k)$-map. Take $p_{0} \in P-\partial P$ and let $W=\varphi^{-1}\left(p_{0}, 0\right)$. If $\varphi=(f, g)$ and $f_{y}:(P, \partial P) \rightarrow$ $(P, \partial P)$ has non-zero degree $\left(\right.$ in $\left.\mathbb{Z}_{2}\right)$, where $f_{y}(x)=f(x, y), x \in P, y \in V_{n, k}$, then

$$
\text { category } \tilde{W} \geq \frac{k(k-1)}{2}+1 \text {, }
$$

where $\tilde{W}$ is the orbit space of $W$ with respect to $T(k)$.

Returning to theorem 5.4, we note that when $k=1$ we have the classical result. It is also interesting to observe that for each value of $k$

$$
\left(t_{1} t_{2} \cdots t_{k}\right)^{n-k+1} \subset \operatorname{Index}{ }^{T(k)} V_{n, k} .
$$

One way to see this is to show that $\left(t_{1} t_{2} \cdots t_{k}\right)^{n-k}$ actually maps onto the topdimensional cohomology class of the manifold $G_{n, k}$ under $H^{*}\left(G_{n, k}\right) \leftarrow H^{*}(B O(k))$ (induced by the classifying map). This shows that our proof fails for $n-k$ increased by one, for any $k$.

Indeed the following example, suggested by a referee, shows that $p=n-k$ is the best possible for a $T(k)$-map $f: V_{n, k} \rightarrow\left(\mathbb{R}^{k}\right)^{p}$ to have zeros. Represent an element of $V_{n, k}$ as a $k \times n$ matrix $A$ and for $1 \leq p \leq n$ let $f: V_{n, k} \rightarrow\left(\mathbb{R}^{k}\right)^{p}$ denote the map which sends $A$ into the last $p$ columns of $A$. Then $f$ is a $G$-map for any subgroup of $O(k)$ and for $p=n-k+1, f$ has no zeros. 
Remark 5.7. If in the preceding results $\mathbb{R}$ is replaced by the complex numbers $\mathbb{C}$ or the quaternions $\mathbb{H}$, then corresponding results obtain. The proofs are essentially identical.

As our final application of the use of the ideal-valued index in obtaining theorems of the Bourgin-Yang type, we mention the following general result. The proof is analogous to the proof of theorem 4.1 with $\delta$ - Index $_{B}^{G}$ replacing Index ${ }^{G}$. It is particularly useful in proving Bourgin-Yang theorems for maps of $G$-sphere bundles to $G$-vector bundles. In particular, the results in [5] which employed the numerical analogues of index theory follow readily using it.

Theorem 5.8. Let $f:(X, A) \rightarrow\left(X^{\prime}, A^{\prime}\right)$ denote a $G$-map over $B$, where $\Lambda=H^{*}(B G)$ is Noetherian and $H^{*}(B)$ is finitely generated over $\mathbb{K}$. Assume $Z^{\prime}$ is a closed G-set in $X^{\prime}$ and $f(A) \subset A^{\prime}-Z^{\prime}$. If

$$
(f \mid A)_{G}^{*}: H_{G}^{*}\left(A^{\prime}-Z^{\prime}\right) \rightarrow H_{G}^{*}(A)
$$

is surjective and $Z=f^{-1}\left(Z^{\prime}\right)$, then

$$
\text { Index }{ }_{B}^{G} Z \subset\left(\delta \text {-Index } B_{B}^{G}(X, A): \delta-I n \operatorname{dex}_{B}^{G}\left(X^{\prime}-Z^{\prime}, A^{\prime}-Z^{\prime}\right)\right) .
$$

6. Index ${ }^{G}(\cdot)$ and the Ljusternik-Schnirelmann method

We close with a brief preview on the use of the ideal-valued index theory in the Ljusternik-Schnirelmann method in critical point theory.

For simplicity, our setting will be a compact $G$-manifold $M$. Let $\Sigma$ denote the family of closed $G$-sets in $M$ and $\Lambda=H^{*}(B G)=H^{*}(B G, \mathbb{K})$, where $\mathbb{K}$ is the field of coefficients, usually suppressed in the notation. $I(M)$ will denote the ideals $\not h$ in $\Lambda$ such that $\operatorname{Index}^{G}(M) \subset \mu$. Then let

$$
\Sigma_{h}=\left\{X \in \Sigma: \text { Index }{ }^{G} X \subset \not h\right\}, \quad \not \in \in I(M) .
$$

Then, if $f: M \rightarrow \mathbb{R}$ is a $C^{1}$-functional, we set

$$
c_{k}=\inf _{x \in \Sigma_{k}} \sup _{x \in X} f(x)
$$

It is not difficult to show that each $c_{\mu}$ is a critical value, called an ideal critical value for $f$. Now let $c$ denote an ideal critical value and $I(c)=\left\{h \in I(M): c_{n}=c\right\}$. Also let $M_{c}=\{x: f(x) \leq c\}$ and $K_{c}$ be the critical set with critical value $c$. Then $h_{*}=$ Index ${ }^{G} M_{c}$ is the minimal ideal in $I(c)$. Furthermore, the multiplicity result on the size of $K_{c}$ takes the following form: there is an ideal $a_{c} \subset \Lambda$ such that:

(1) $a_{c} \supsetneq h_{*}$;

(2) $a_{c} \not \subset \not h, h \in I(c)$;

(3) Index ${ }^{G} K_{c} \subset\left(\not_{*}: a_{c}\right)$.

Under certain conditions this forces $K_{c}$ to have positive $G$-cohomology. Finally, if $M$ admits a proper filtration of ideals

$$
\Lambda \supset \hat{\Lambda}=h_{1} \supset h_{2} \supset \cdots \supset \mu_{N}=\operatorname{Index}^{G} M
$$

with an appropriate property, then any $C^{1} G$-map $f: M \rightarrow \mathbb{R}$ has at least $N$ critical orbits. This suggests a 'Morse theory' based upon ideal-valued index theory and this will be the subject of a forthcoming article. 
Acknowledgement. Both authors were supported in part by the National Scientific Foundation under grant \#DMS-8320099.

\section{REFERENCES}

[1] M. Atiyah \& I. G. Macdonald. Introduction to Commutative Algebra. Addison-Wesley, Reading, MA (1969).

[2] G. E. Bredon. Introduction to Compact Transformation Groups. Academic Press, New York (1972).

[3] A. Dold. Lectures on Algebraic Topology. Springer-Verlag, New York (1972).

[4] E. Fadell \& S. Husseini. Relative cohomological index theories. Adv. Math. To be published.

[5] E. Fadell \& S. Husseini. Index theory for $G$-bundle pairs with applications to Borsuk-Ulam type theorems for $G$-sphere bundles. Non-Linear Functional Analysis, ed. G. Rassias.

[6] E. Fadell \& P. Rabinowitz. Generalized cohomological index theories for Lie group actions with an application to bifurcation questions for Hamiltonian systems. Invent. Math. 45 (1978), 139-174.

[7] S. Husseini, J-M. Lasry \& M. Magill. Existence of equilibrium with incomplete markets. To appear.

[8] E. Spanier. Algebraic Topology. McGraw-Hill, New York (1966). 